\title{
Is SARS-CoV-2 Transmitted Through Breastfeeding?
}

\section{S. Thanigainathan ${ }^{1} \cdot$ Venkatesh Kaliyaperumal $^{2} \cdot \operatorname{Sindhu}_{\text {Sivanandan }}{ }^{1} \cdot$ Sasirekha Rengaraj $^{3} \cdot$ Rahul Dhodapkar $^{2}$. Adhisivam Bethou ${ }^{1}$ (B)}

Received: 15 October 2020 / Accepted: 25 January 2021 / Published online: 8 February 2021

(C) Dr. K C Chaudhuri Foundation 2021

\begin{abstract}
There are concerns regarding the transmission of severe acute respiratory syndrome coronavirus 2 (SARS-CoV-2) from mother to child during this COVID pandemic. This descriptive study was done to check the possible transmission of the virus through breastfeeding in the Indian context. RT-qPCR for SARS-CoV-2 was done in breast milk samples from 30 COVID-positive mothers. Paired oropharyngeal swabs of the same neonates were also sent for RT-PCR at $48 \mathrm{~h}$ and on day 5 of life. All the breast milk samples were negative for SARS-CoV-2 except one. A repeat sample of breast milk from the same mother was also negative when rechecked the next day. All the paired neonatal oropharyngeal swabs were also negative for SARS-CoV-2. The authors could not find evidence for transmission of SARS-CoV-2 from mother to child through breastmilk in the population studied.
\end{abstract}

Keywords COVID-19 $\cdot$ Breast milk $\cdot$ SARS-CoV-2 $\cdot$ Pandemic

\section{Introduction}

Exclusive breastfeeding during coronavirus disease (COVID-19) pandemic is vital for survival of neonates. However, there are concerns regarding the transmission of severe acute respiratory syndrome coronavirus 2 (SARS-CoV-2) from mother to child through breast milk. Viral RNA detection in breast milk samples from few women infected with SARS-CoV-2 have been reported [1-3]. Evidence is emerging supporting breastfeeding but there is paucity of Indian data in this area. This study was done to check the possible transmission of the virus through breastfeeding in the Indian context.

Adhisivam Bethou

adhisivam1975@yahoo.co.uk

1 Department of Neonatology, Jawaharlal Institute of Postgraduate Medical Education and Research (JIPMER), Pondicherry 605 006, India

2 Department of Microbiology, Jawaharlal Institute of Postgraduate Medical Education and Research (JIPMER), Pondicherry, India

3 Department of Obstetrics and Gynecology, Jawaharlal Institute of Postgraduate Medical Education and Research (JIPMER),

Pondicherry, India

\section{Methods}

This descriptive study was done in a tertiary care teaching hospital in south India after due approval from the Institute Ethics Committee (JIP/IEC/2020/061). Mothers admitted for delivery were tested for SARS-CoV-2 infection by reverse transcription polymerase chain reaction (RT-PCR) of throat swabs. After due informed consent, breast milk samples from 30 COVIDpositive mothers admitted consecutively were collected between 48 to $72 \mathrm{~h}$ following delivery. The milk samples were collected by manual expression using aseptic techniques. Detection of viral loads using RT-qPCR for SARS-CoV-2 in whole breast milk was done using WHO recommended primer probe sequence for SARS-CoV-2 Orfl $1 b$ gene followed by confirmation with SD biosensor RT-PCR for $R d R p$ and $E$ gene. Paired oropharyngeal swabs of the same neonates were also sent for RT-PCR at $48 \mathrm{~h}$ and on day 5 of life. The motherbaby dyads were kept together and mothers exclusively breastfed their babies. Use of masks, appropriate hand hygiene and disinfection of frequently touched surfaces were practiced by the mothers. The healthy mother-baby dyads were discharged after $10 \mathrm{~d}$.

\section{Results}

There were 3198 deliveries in the hospital from April to August, 2020. COVID positivity among delivering mothers 
was $0.14 \%, 3.3 \%$, and $7.8 \%$ during the months of June, July, and August, respectively. All the breast milk samples were negative for SARS-CoV-2 except 1. A repeat sample of breastmilk from the same mother was also negative when rechecked the next day. All the paired neonatal oropharyngeal swabs were also negative for SARS-CoV-2. No clinical manifestations of COVID was noted among the neonates.

\section{Discussion}

Though the COVID positivity among delivering mothers increased proportionate to that of the general population, their neonates were not affected. Moreover, the authors could not find evidence for transmission of SARS-CoV-2 from mother to child through breast milk in the population studied. Even the odd breast milk sample which was initially positive could be due to contamination or just evidence of nonreplicating virus. Recent studies from Wuhan, China have also shown similar findings $[4,5]$. These results are reassuring and all postnatal mothers irrespective of their COVID status should be encouraged to exclusively breastfeed their babies and follow general safety precautions. Moreover, SARS-CoV-2reactive antibodies in breast milk may also provide passive immunity to breastfed infants and protect them against COVID-19 [6].

Acknowledgements The authors want to thank the pediatric postgraduates and DM Neonatology fellows who helped in sample collection.
Authors' Contributions ST designed the study and coordinated sample collection; VK and RD helped in RT-PCR; SS coordinated clinical management; SR coordinated management of mothers; AB supervised the study, drafted manuscript and will act as guarantor for this paper.

\section{Declarations}

Conflict of Interest None.

\section{References}

1. Groß R, Conzelmann C, Müller JA, et al. Detection of SARS-CoV-2 in human breastmilk. Lancet. 2020;395(10239):1757-8.

2. Chambers C, Krogstad P, Bertrand K, et al. Evaluation for SARSCoV-2 in breast milk from 18 infected women. JAMA. 2020;19: e2015580. https://doi.org/10.1001/jama.2020.15580.

3. Costa S, Posteraro B, Marchetti S, et al. Excretion of SARS-CoV-2 in human breast milk. Clin Microbiol Infect. 2020. https://doi.org/10. 1016/j.cmi.2020.05.027.

4. Chen $\mathrm{H}$, Guo J, Wang C, et al. Clinical characteristics and intrauterine vertical transmission potential of COVID-19 infection in nine pregnant women: a retrospective review of medical records. Lancet. 2020;395(10226):809-15.

5. Liu W, Wang J, Li W, Zhou Z, Liu S, Rong Z. Clinical characteristics of 19 neonates born to mothers with COVID-19. Front Med. 2020;14(2):193-8.

6. Demers-Mathieu V, Dung M, Mathijssen GB, et al. Difference in levels of SARS-CoV-2 S1 and S2 subunits- and nucleocapsid protein-reactive SIgM/IgM, IgG and SIgA/IgA antibodies in human milk [published online ahead of print, 2020 Sep 1]. J Perinatol. 2020. https://doi.org/10.1038/s41372-020-00805-w.

Publisher's Note Springer Nature remains neutral with regard to jurisdictional claims in published maps and institutional affiliations. 